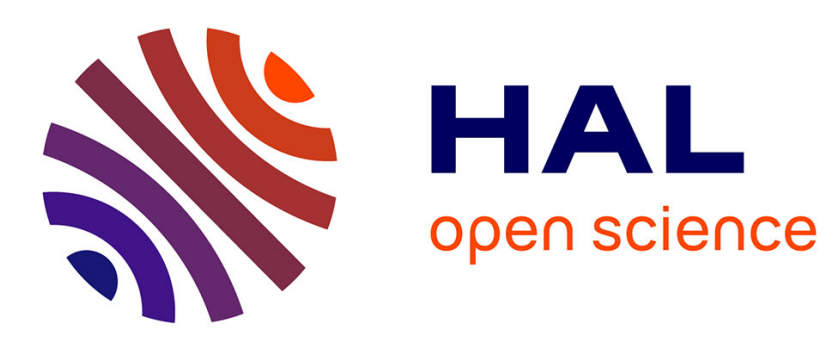

\title{
Crues et inondations en Roussillon : le risque, le discours et l'aménagement.
}

Bertrand Desailly

\section{To cite this version:}

Bertrand Desailly. Crues et inondations en Roussillon : le risque, le discours et l'aménagement.. Revue Géographique des Pyrénées et du Sud-Ouest, 1990, 61 (4), pp.515-519. 10.3406/rgpso.1990.3229 . hal-03161047

\section{HAL Id: hal-03161047 \\ https://hal.science/hal-03161047}

Submitted on 5 Mar 2021

HAL is a multi-disciplinary open access archive for the deposit and dissemination of scientific research documents, whether they are published or not. The documents may come from teaching and research institutions in France or abroad, or from public or private research centers.
L'archive ouverte pluridisciplinaire HAL, est destinée au dépôt et à la diffusion de documents scientifiques de niveau recherche, publiés ou non, émanant des établissements d'enseignement et de recherche français ou étrangers, des laboratoires publics ou privés. 


\section{Crues et inondations en Roussillon : le risque, le discours et} l'aménagement

\section{Bertrand Desailly}

\section{Citer ce document / Cite this document :}

Desailly Bertrand. Crues et inondations en Roussillon : le risque, le discours et l'aménagement. In: Revue géographique des Pyrénées et du Sud-Ouest, tome 61, fascicule 4, 1990. Forêts des Pyrénées. pp. 515-519;

doi : https://doi.org/10.3406/rgpso.1990.3229

https://www.persee.fr/doc/rgpso_0035-3221_1990_num_61_4_3229

Fichier pdf généré le 06/04/2018 
REVUE GÉOGRAPHIOUE DES PYRÉNÉES ET DU ST(1)-OUEST TOME 61, FASC. 4, pp. 515-528, Toulouse, 1990.

\title{
CHRONIQUE
}

\author{
CRUES ET INONDATIONS EN ROUSSILLON : \\ LE RISOUE, LES DISCOURS ET L'AMENAGEMENT
}

\begin{abstract}
A l'image de bon nombre de contrées méditerranéennes, le Roussillon est de temps à autre affecté par des crues et des inondations catastrophiques. On songe naturellement à l'aiguat des 16 et 17 octobre 1940, qui fit une quarantaine de victimes dans la vallée du Tech et causa des dégâts énormes de part et d'autre du Canigou. Un colloque scientifique s'est récemment tenu dans la station thermale de Vernet-les-Bains, afin de marquer - on n'oserait dire célébrer - le cinquantième anniversaire de l'événement (1).
\end{abstract}

La crue d'octobre 1940 parce qu'elle a atteint dans l'est des Pyrénées une ampleur tout à fait exceptionnelle - son temps de retour serait de l'ordre de plusieurs siècles - a logiquement cristallisé les curiosités les plus variées. Mais peut-être au point de faire négliger d'autres formes de recherches prenant en compte la moyenne ou la longue durée historique. Un simple examen des relevés de hauteurs d'eau effectués régulièrement à Perpignan depuis 1876 montre que la Têt y a atteint ou dépassé à quatre reprises, hormis en 1940, la cote de quatre mètres, qui correspond à un débit égal ou supérieur à $1500 \mathrm{~m}^{3} / \mathrm{s}$. Durant quelques jours, en 1876, 1892, 1898 et 1920, la plaine côtière de la Salanque cut à souffrir de très graves inondations Et pas plus qu'elle n'a mis fin soudainement à une longue période de tranquillité, la catastrophe d'octobre 1940 n'a fait surgir en Roussillon les premières politiques cohérentes de défense contre les eaux. Dès le Moyen Age, les riverains de la Têt étaient organisés

(1) Les inondations d'octobre 1940 en Catalogne. 50 ans passés. Colloque organisé par le Centre de recherche et d'études catalanes (Université de Perpignan) et par le Centre de géographie physique Henri Elhaï (Université de Paris X), Vernet-les-Bains, 18-20 octobre 1990. 
en associations, qui s'efforçaient de consolider les berges du fleuve grâce a des plantations.

Or, les archives sont susceptibles de fournir de précicuses informations à la fois sur le déroulement de quelques grancles crues du passé et sur les moyens de prévention employés à différentes époques. On découvrira par exemple à l'intérieur de la série $C$ des Archives départementales des Pyrénées-Orientales plusieurs centaines de pièces relatives à des travaux effectués dans le lit de la Tét avant la Révolution. Il s'agit pour une large part de mémoires et de rapports rédigés par des ingénieurs du Génie militaire ou des Ponts et Chaussées, accompagnés parfois de plans ou de profils. Bien entendu, on ne peut attendre de telles sources des renseignements précis concernant les débits maximums atteints par les cours d'eau L'extension spatiale des grands aiguats du XVIII siècle nous est cependant assez bien connue, grâce aux états d'indemnités octroyées à ces occasions aux différentes communautés. Surtout, l'ensemble de ces textes fournit la matière à une réflexion sur la genèse et le déroulement des processus d'aménagement.

Notre propos a été de mettre en évidence, sur le long terme historique. quelques grands moments caractérisés par l'apparition de certains discours sur le risque, par la mobilisation dans la défense contre les eaux de certains groupes sociaux - ingénieurs, syndicats de riverains, notables locaux -, par la primauté enfin accordée à certaines formes d'interventions (2). Pour des raisons tenant à la constitution des sources utilisées, l'intervalle de temps retenu a été de deux siècles et demi, soit du rattachement en 1659 de la province du Roussillon au Royaume de France jusqu'au milieu du $\mathrm{XX}^{\mathbf{e}}$ siècle.

De 1660 à 1750 environ, les seuls ouvrages importants de protection contre les eaux édifiés en Roussillon sont le fait des ingénieurs du Génie militaire. Ceux-ci n'interviennent que de façon ponctuelle, dans les lieux présentant un intérêt stratégique. Leur mission principale est en effet, dans la perspective d'une reprise possible des hostilités avec l'Espagne, d'assurer le maintien en état des places-fortes de la province, au premier rang desquelles se trouve Perpignan L'endiguement de la Têt dans sa traversée de la ville, achevé en 1730, s'inscrit directement dans cette perspective. Il s'agit de protéger de l'irruption des eaux les glacis des fortifications, mais aussi de tirer parti de la présence d'un fleuve capricieux pour gêner l'organisation d'un siège (fig. 1).

C'est dans les dernières décennies du XVIII" siècle que le discours sur les crues et les moyens de s'en préserver prend un tour résolument moderne. On le doit aux ingénieurs des Ponts et Chaussées qui opèrent dans la généralité à partir de 1750 . Concrètement, les défrichements pra-

(2) Bertrand DEsaILly, Crues et inondations en Roussillon. Le risque et l'aménagement. Fin du XVII' siècle-milieu du XX $X^{\circ}$ siècle, $1990,352 \mathrm{p.,}$ fig., biblio. (Thèse de doctorat de Géographie, Université de Paris X-Nanterre.) 
tiqués dans la montagne sont rendus responsables de l'aggravation des inondations dans la plaine voisine. De tels propos sont sous-tendus par une perception largement nouvelle des interactions spatiales - entre la plaine et les hauts pays, et par la diffusion du concept de bassin hydrographique, à la suite des écrits du géographe Philippe Buache (3). La pratique de l'aménagement demeure cependant en retrait sur le discours, probablement parce que les ingénieurs demeurent prisonniers de certaines contradictions. La condamnation des essartages sur les pentes s'accommode assez mal des idées propagées par les physiocrates. Les membres du corps des Ponts et Chaussées demeurent par ailleurs fortement attachés à la réalisation d'édifices monumentaux tels que les digues ou les ponts, socialement valorisants, alors que le contrôle des écoulements sur les versants ravinés exige la dissémination d'une multitude de petits ouvrages, qui plus est dans une montagne nord catalane alors peu fréquentée par les gens de qualité.

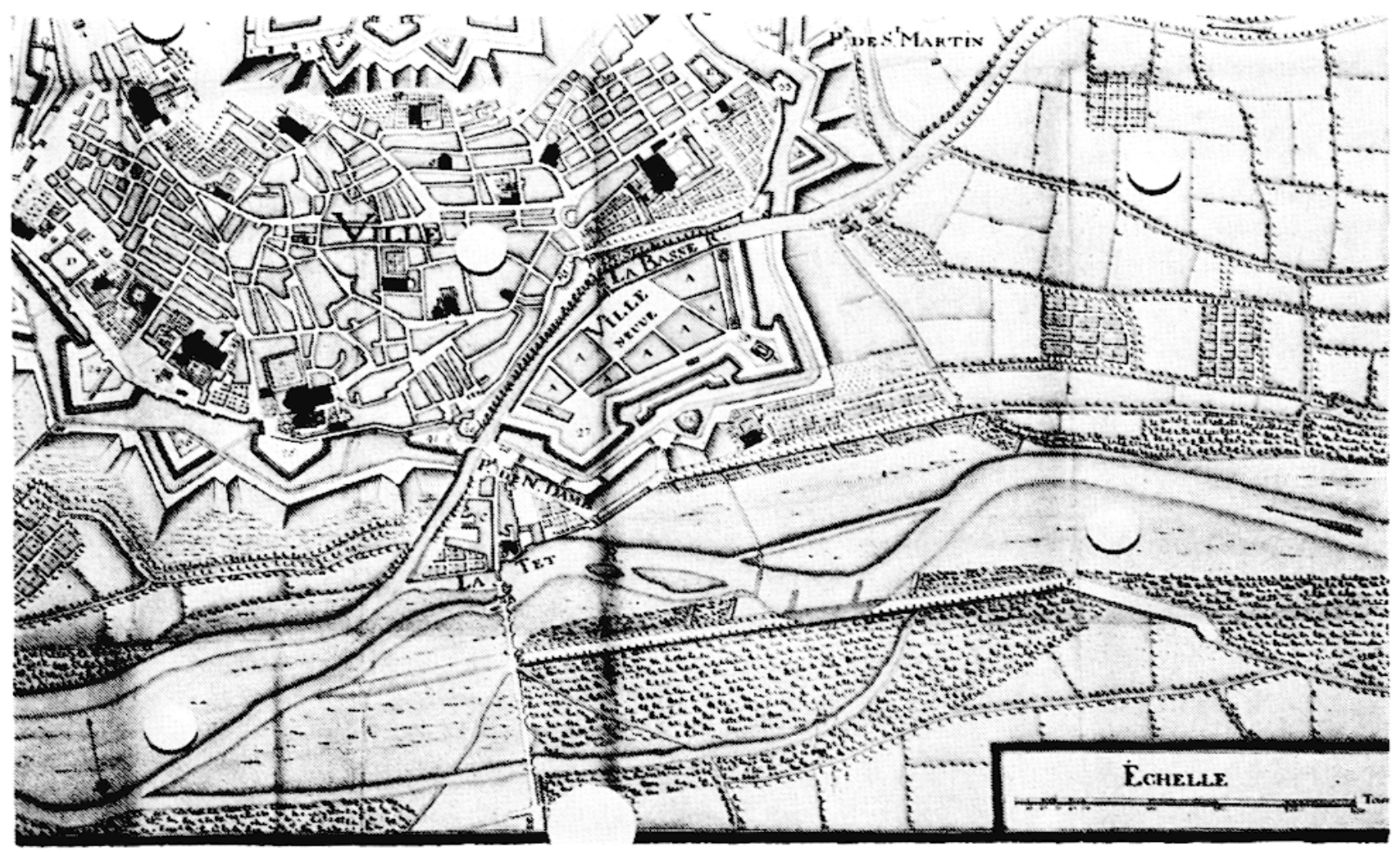

Fug. 1

La Têt à Perpignan après la crue du 12 novembre 1737. (Le plan est orienté vers le sud.)

Les digues édifiées dix ans auparavant par les ingénieurs du Génie viennent de montrer leur fragilité. Epis et plantations n'ont pu empćcher l'ouverture d'un brèche dans l'ouvrage de rive gauche.

(Source: Archives de l'Inspection du Génie, article 8, section 1, Perpignan.)

(3) Philippe Buache, «Essai de géographie physique... », Mémoires de l'Académie royale des Sciences, 1752, pp. 399-416. 
Le dernier quart du $\mathrm{XIX}^{\bullet}$ siècle enfin voit la mise en ceuvre des conceptions en matière de lutte contre les crues qui étaient en germe cent ans plus tôt. Sous la Restauration et la Mcnarchie de Juillet, cellesci avaient rencontré un écho favorable parmi les élites locales, réunies au sein de sociétés savantes (4). Ces aspects sont généra'ement mieux connus, qu'il s'agisse de la constitution de périmètres de restauration des terrains en montagne à la suite des trois lois de 1860, 1864 et 1885 (5), ou bien de l'organisation à partir de 1876 de services spéciaux d'annonce des crues, permise par la diffusion du télégraphe On insistera seulement, à propos du premier point, sur la persistance de relations confllictuelles entre les responsables de la R.T.M. et les populations des montagnes roussillonnaises. Alors que dans les Alpes et les Pyrénées centrales, les tensions vives des débuts se sont un peu relâchées à l'aube du $\mathbf{X X}^{\circ}$ siècle, le 12 février 1905 se tient encore dans la petitc ville d'Olette un " congrès contre le reboisement ", réunissant les maires et conseillers des communes du haut Conflent.

Le principal enseignement de cette étude est la relative autonomie entre la chronologie des crues catastrophiques dans les petits bassins fluviaux des Pyrénées orientales, l'évolution des discours sur le risque et les transformations des pratiques de l'aménagement. 1940, pas plus que 1892 ou 1920, années où la Têt, l'Agly et le Tech connurent de très grands débordements, ne constituent à proprement parler des dates charnières dans l'histoire des politiques de gestion du risque naturel dans l'Est des Pyrénées. Certes, quelques événements ont bien fourni le prétexte à la mise en pratique d'idées neuves. Il en est ainsi sous l'Ancien Régime des inondations de juin, novembre et décembre 1777, qui furent suivies un an plus tard d'une ordonnance de l'intendant Raymond de Saint-Sauveur interdisant tout défrichement sur les hauteurs de la province. De même à l'échelle nationale, les inondations très étendues du printemps 1856 fournirent à l'administration des forêts l'occasion de fairc voter, quatre ans après, la première loi sur le reboisement des montagnes. Mais dans chaque cas, les décisions rendues ne constituaient que l'aboutissement de réflexions qui avaient pris forme bien avant que ne surviennent ces épisodes hydrauliques extraordinaires. La crue d'octobre 1940, parce qu'elle survint à l'issue d'une période d'entre-deux-guerres marquées par un certain manque d'imagination en matière de défense contre les eaux, n'a pas engendré de renisc en question comparable.

Pas plus qu'elle ne se calque vraiment sur les rythmes catastrophiques la succession des différents épisodes de la lutte contre les crues en Roussillon ne constitue un reflet fidèle et exclusif des changements d'ordre politique, ou bien de l'évolution des mentalités, qui ont carac-

(4) Notamment, en Roussillon, la Société agricole, scientifique et littéraire des Pyrénées-Orientales, fondée en 1833.

(5) Cf. Louis DE CRÉcy, L'histoire de la R.T.M.: quelques réflexions d'un praticien, RGPSO, tome 59,1988 , pp. 19-22. 
térisé les trois derniers siècles. Sans doute, le premicr endiguement de la Têt à Perpignan, tel que l'ont conçu peu après 1700 les ingénieurs militaires, est étroitement lié aux conditions geopolitiques de l'époque. Inversement, on serait bien en pleine d'attribuer à l'épisode révolutionnaire un influence notable sur l'attitude des ingénieurs face aux débordements des cours d'eau. Au total, on se trouve donc en présence d'une histoire singulièrement complexe, dont les clés sont multiples. On ne saurait en particulier passer sous silence l'influence des modèles techniques et culturels propres à chaque époque. La volonté exprimée au XVIII' siècle d'appliquer coûte que coûte aux petits flcuves roussillonnais de grands alignements droits, afin de mettre un terme à leurs divagations, témoigne aussi de la large diffusion de normes esthétiques qui sont au fond celles du jardin à la française, et qui prévalent alors dans le tracé des nouvelles routes ou des canaux.

Bertrand DESAILI,Y.

\section{LES AVATARS D'UNE FORET DE BIGORRE DEPUIS LE XI' SIECLE}

Au nord de la bourgade de Saint Pé de Bigorre, installés dans une vallée aux flancs raides creusés dans les flyschs cénomaniens, se trouvent les 70 hectares du canton "Bédat * de la forêt domaniale indivise de Saint Pé. Si le versant est en est aujourd'hui enrésiné en pin laricio de Corse, le versant ouest doit paraitre à l'observateur, quant à sá composition en essences, proche d'un état originel sans intervention humaine. L'examen des archives montre qu'il n'en est rien et que ce canton forestier a subi en dix siècles d'histoire plusieurs bouleversements.

\section{Des origines au $X I^{\bullet}$ siècle.}

A notre connaissance, le premier témoignage écrit mentionnant ce canton date de 1022 : c'est l'acte de donation en toute propriété du Bédat aux religieux de Saint Pé par Sanche $V$, prince et duc de Gascogne, qui correspond à la fondation de l'abbaye (6).

(6) A la Révolution, ce bien religieux devenu bien des Domaines de l'Etat restait grevé d'importants droits d'usage au profit des habitants de la commune de Saint Pé : cette lourde servitude fut assimilée en 1827 à une copropriété à parts égales de $50 \%$. 